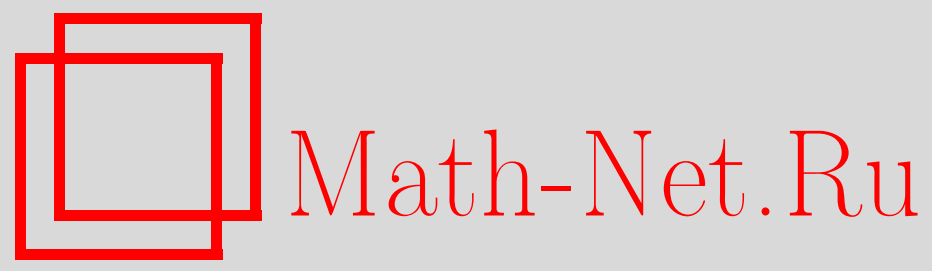

А. И. Никонов, М. Ю. Антонов, Об отсчётах парных функций экспертной оценки, Вестн. Сам. гос. техн. ун-та. Сер. Физ.-мат. науки, 2012, выпуск 2(), 158163

DOI: https://doi.org/10.14498/vsgtu1054

Использование Общероссийского математического портала Math-Net.Ru подразумевает, что вы прочитали и согласны с пользовательским соглашением

http://www.mathnet.ru/rus/agreement

Параметры загрузки:

IP : 34.227 .88 .159

26 апреля 2023 г., 14:50:20

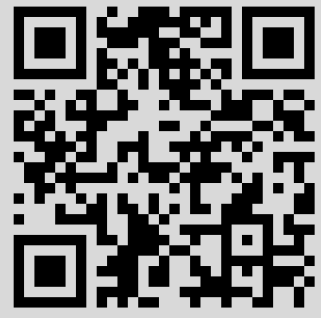


Вестн. Сам. гос. техн. ун-та. Сер. Физ.-мат. науки. 2012. № 2 (27). С. $158-163$

\title{
Вычислительная математика
}

\section{УДК 519.584}

\section{ОБ ОТСЧЁТАХ ПАРНЫХ ФУНКЦИЙ ЭКСПЕРТНОЙ ОЦЕНКИ}

\author{
М. Ю. Антонов, А.И. Никонов \\ Самарский государственный технический университет, \\ 443100, Россия, Самара, ул. Молодогвардейская, 244. \\ E-mails: makcant@yandex.ru, nikonovai@mail.ru
}

\begin{abstract}
В рассмотрение введены парные функиии, используемые применительно к объекту стоимостной экспертной оценки, который участвует вместе с рядом других объектов в реализации системы производственного или исследовательского назначения. Эти функиии, имеющие согласованный характер возрастания в отношении критерия качества данного объекта, образуют оценочную математическую модель. Доказано наличие однозначности, проявляемое отсчётами функций рассматриваемого вида в условиях воздействия фактора времени. Признаки такого проявления связаны с заданием определённых знаков частных приращений рассматриваемых функиий, соответствующих изменениям критерия качества очениваемого объекта, а такюе временной задержке момента его приобретения сравнительно с моментом выдачи экспертной информации.
\end{abstract}

Ключевые слова: экспертная оценка, обгект оценки, функция экспертной оценки, согласованные парные функиии, отсчёт значений.

Цель настоящей работы заключается в выявлении важного свойства функций экспертной оценки (ЭО) - однозначности отсчёта пары функций, представляющей выходную экспертную информацию в условиях возможного сдвига временных моментов ее формирования и использования. Предметом проведения экспертизы является стоимость отдельно взятого технического объекта, входящего в спроектированную систему производственного или исследовательского назначения. Заказчиком проведения экспертизы выступает организация-разработчик проекта данной системы; эту организацию представляют, в частности, е' руководитель, а также ведущий специалист, ответственный за состояние проекта. Наличие указанного свойства оказывается полезным для обоснования определенности и выработки контрольной информации стоимостной экспертной оценки.

Функция ЭО задается взаимно-однозначным соответствием критерия качества $X$-совокупного показателя, относящегося к заданному множеству характеристик объекта оценки (ОбО), и значений вида $y=f_{\ni}(X)$, которые принадлежат шкале, содержащей дискретное множество собственно оценочных уровней, для введенной нами определенности - уровней стоимостной оценки. Критерий $X$ изменяется непрерывно. Такая функция представляет со-

Максим Юръевич Антонов, аспирант, каф. электронных систем и информационной безопасности. Александр Иванович Никонов (д.т.н., проф.), профессор, каф. электронных систем и информационной безопасности. 
бой одну из основных частей оценочной математической модели. Поскольку в сфере распространения интересующих нас системных объектов наблюдаются реальные случаи как увеличения, так и уменьшения их стоимости, естественным является введение в оценочную математическую модель пары функций ЭО $f_{\ni 1}(X), f_{\ni 2}(X)$, которые должны быть согласованы друг с другом, то есть характерные изменения этих функций на участках согласования должны быть сходны между собой. Парные функции, относящиеся к функциям непрерывного вида [1], использовались ранее при описании некоторых объектов измерительной техники [2]. Диапазон практически приемлемых изменений критерия $X$ обозначим через $D_{X}$, а общую для $f_{\ni 1}(X), f_{\ni 2}(X)$ область значений - через $D_{y}=\left[y_{\mathrm{H}}, y_{\mathrm{B}}\right] ; y_{\mathrm{H}}, y_{\mathrm{B}}$ - положительные дискретизированные значения, нижняя и верхняя границы шкалы. Функции $f_{\ni 1}(X), f_{\ni 2}(X)$ можно рассматривать как дискретные аналоги соответствующих монотонных функций $f_{\sim 1}(X), f_{\sim 2}(X)$, проходящих через точки на графиках вида $\left(y_{1}, X\right)$, $\left(y_{2}, X\right)$

$$
y_{1}=f_{\ni 1}(X), y_{2}=f_{\ni 2}(X) .
$$

Функции $f_{\sim 1}(X), f_{\sim 2}(X)$ могут быть построены, в частности, как известные интерполяционные полиномы [3]. Уровни величин $X$ складываются под влиянием факторов вида $\chi_{i}$, характеризующих различные аспекты состояния ОбО:

$$
\begin{gathered}
X=\sum_{i=1}^{n} \alpha_{i} \chi_{i}, \chi_{i} \in\left[\chi_{i \mathrm{H}}, \chi_{i \mathrm{~B}}\right], \sum_{i=1}^{n} \alpha_{i}=1 ; \\
X \in D_{x}=\left[X_{\mathrm{H}}, X_{\mathrm{B}}\right] .
\end{gathered}
$$

Считаем, что возрастанию значений критерия $X$ соответствует возрастание значений парных согласованных функций $f_{\ni 1}(X), f_{\ni 2}(X)$, однако при этом значения вида $y_{1}$ соответствуют ситуации возрастания стоимости, а значения вида $y_{2}-$ ситуации ее убывания во времени $t ; t \in\left[0, t_{\mathrm{B}}\right]=D_{t}$. Момент $t=0$ соответствует моменту поступления информации от экспертной группы. Как видим, рассматриваемые парные функции являются, в сущности, функциями двух переменных $(X, t)$, где время представляет собой дополнительный аргумент. Каждая из функций $f_{\sim j}\left(X, t^{*}\right)=f_{\sim j t^{*}}(X), f_{\sim j}\left(X^{*}, t\right)=$ $=f \sim X^{*}(t)$ имеет обратную; здесь через $X^{*}$ и $t^{*}$ обозначены фиксированные уровни аргументов. Вместе с получением оценки стоимости данного технического объекта заказчик ожидает получить от экспертной группы прогноз изменения этой стоимости в заданном интервале $D_{t}$.

Итак, предметом нашего исследования являются согласованные парные функции вида $f_{\ni j}(X, t)$, которые при $X \in D_{X}, t \in D_{t}, y_{j} \in D_{y}(j=1,2)$ образуют именно модель оценки отдельно взятого системного объекта. При необходимости количество оцениваемых объектов, каждый из которых обладает собственным набором количественных характеристик и которому соответствует собственная пара функций ЭО, можно наращивать вплоть до их заданного конечного числа. Установим справедливость нижеприводимого утверждения, отражающего свойство однозначности отсчётов парных согласованных функций ЭО со значениями в дискретизированном числовом множестве.

Пусть имеется пара функций $f_{\ni 1}(X, t)$ и $f_{\ni 2}(X, t)$, аргументы которых непрерывны, а значения принадлежат дискретизированному диапазону $D_{y}$. 
Пусть заданы следующие знаки разностных аналогов производных $\partial f_{\sim j} / \partial X$, $\partial f_{\sim j} / \partial t:$

$$
\left.f_{\ni j X}^{\prime}\right|_{t^{*}}=\left.\triangle f_{\ni j X}\right|_{t^{*}} / \triangle X_{j l}=h / \triangle X_{j l}>0
$$

где

$$
\begin{gathered}
\triangle X_{j l}=X_{j l+1}-X_{j l}, \quad X_{j l}=\left.f_{\sim j Y}^{-1}\right|_{t^{*}}\left(f_{\sim j l}\right), \quad X_{j l+1}=\left.f_{\sim j Y}^{-1}\right|_{t^{*}}\left(f_{\sim j l+1}\right), \\
f_{\sim j l}=l_{j} h, \quad f_{\sim j l+1}=\left(l_{j}+1\right) h, \quad j=1,2
\end{gathered}
$$

и

$$
\begin{aligned}
& \left.f_{\ni 1 t}^{\prime}\right|_{X^{*}}=\left.\triangle f_{\ni 1 t}\right|_{X^{*}} / \triangle t_{1 l}=h / \triangle t_{1 l}>0, \\
& \left.f_{\ni 2 t}^{\prime}\right|_{X^{*}}=\left.\triangle f_{\ni 2}\right|_{X^{*}} / \triangle t_{2 l}=-h / \triangle t_{2 l}<0,
\end{aligned}
$$

где

$$
\triangle t_{j l}=t_{j l+1}-t_{j l}>0, \quad t_{j l}=\left.f_{\sim j Y}^{-1}\right|_{X^{*}}\left(f_{\sim j l}\right), \quad t_{j l+1}=\left.f_{\sim j Y}^{-1}\right|_{X^{*}}\left(f_{\sim j l+1}\right) .
$$

Здесь через $\left.f_{\sim j Y}^{-1}\right|_{t^{*}}\left(f_{\sim j l}\right),\left.f_{\sim j Y}^{-1}\right|_{X^{*}}\left(f_{\sim j l}\right),\left.f_{\sim j Y}^{-1}\right|_{t^{*}}\left(f_{\sim j l+1}\right),\left.f_{\sim j Y}^{-1}\right|_{X^{*}}\left(f_{\sim j l+1}\right)$ обозначены функции, обратные к $f_{\sim j}\left(X, t^{*}\right)$ и $f\left(X^{*}, t\right)$, аргументами в которых выступают соответственно $f_{\sim j l}, f_{\sim j l+1}$, а через $l$ обозначен целочисленный индекс количества шагов дискретизации $D_{y}$, соответствующих тому или иному значению; $y_{\ni}=\left(l-l_{\min }\right) h$ - нижняя граница $\left(l-l_{\min }\right)$-того деления $y_{\ni}$. Символ $l_{j}$ указывает на то, что здесь берётся значение $l$, которое соответствует фиксированному значению аргументов $j$-той функции от $X$ или $t$ и конкретизируется далее как $l_{j}\left(X^{*}, t\right)$ или $l_{j}\left(X, t^{*}\right)$.

Тогда любой аргумент $X \in D_{X}$, для которого произведен отсчёт значений из $D_{y}$, принадлежащих данной паре функций, является единственным в $D_{X}$.

Начиная доказательство этого утверждения, предположим, что оно неверно и, следовательно, отсчёт значений в данной паре функций при любом $X^{*} \in D_{X}$ может оказаться не единственным. Пусть при некоторых значенияX

$$
X=X^{*}, f_{\ni 1}\left(X^{*}, t\right), f_{\ni 2}\left(X^{*}, t\right)
$$

исходное значение $t^{*} \in D_{t}$ получило положительное приращение:

$$
t=t^{*}+\triangle t, \triangle t>0
$$

Заметим также, что разность $\triangle X_{j l}$ является положительной.

Далее, учитывая заданные знаки разностных аналогов производных $\partial f_{\sim 1} / \partial t$ и $\partial f_{\sim 2} / \partial t$, имеем для соответствующих приращений функций $\left.f_{\ni 1}(t)\right|_{X^{*}},\left.f_{\ni 2}(t)\right|_{X^{*}}$ :

$$
\begin{aligned}
& \triangle f_{\ni 1}\left(X^{*}, t\right)=\left.\sum_{\ell=\ell_{1}\left(X^{*}, t^{*}\right)}^{\ell_{1}\left(X^{*}, t\right)-1} f_{\ni 1 t}^{\prime}\right|_{X^{*}} \cdot \Delta t_{1 l}>0 ; \\
& \triangle f_{\ni 2}\left(X^{*}, t\right)=\left.\sum_{\ell=\ell_{2}\left(X^{*}, t\right)}^{\ell_{2}\left(X^{*}, t\right)-1} f_{\ni 2 t}^{\prime}\right|_{X^{*}} \cdot \triangle t_{2 l}<0 .
\end{aligned}
$$


Наличие приращений

$$
\begin{aligned}
& \triangle f_{\ni 1}\left(X^{*}, t\right)=y_{1}\left(X^{*}, t\right)-y_{1}\left(X^{*}, t^{*}\right), \\
& \triangle f_{\ni 2}\left(X^{*}, t\right)=y_{2}\left(X^{*}, t\right)-y_{2}\left(X^{*}, t^{*}\right)
\end{aligned}
$$

иллюстрируется на рисунке, где уровни вида $y_{1}, y_{2}$ представлены наложенными на графики непрерывных вариантов рассматриваемых функций. Штрихи, пересекающие графики вида $y(X)$, указывают на используемые в нашем доказательстве дискретизированные зависимости вида $f_{\ni j}(X)$.

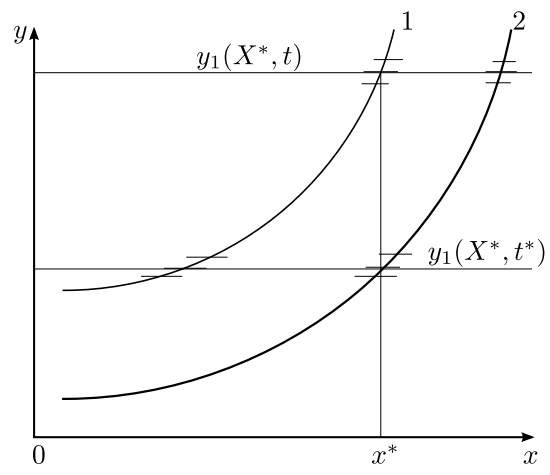

a

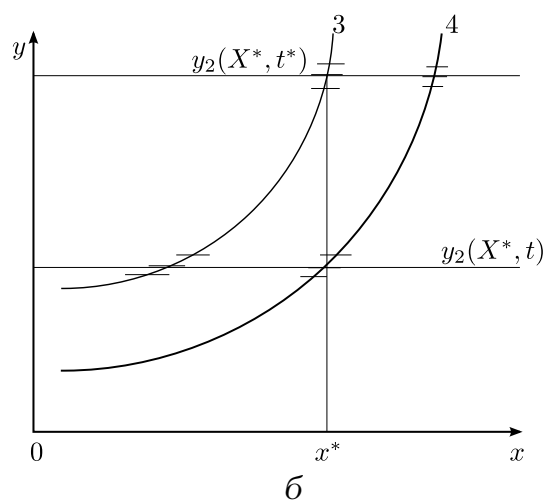

Приращения первой (а) и второй (б) функций: $1-f_{\sim 1}(X, t), 2-f_{\sim 1}\left(X, t^{*}\right), 3-f_{\sim 2}\left(X, t^{*}\right), 4-f_{\sim 2}(X, t)$

Через $X_{t}$ обозначим значение величины $X$, альтернативное $X^{*}$ при возникновении ситуации неединственности отсчёта; $X_{t}=X^{*}+\triangle X_{t}$, где $\triangle X_{t}-$ приращение величины $X$, относимое к изменению времени сравнительно с его исходным моментом $t^{*}$. Согласно нашему исходному предположению о неоднозначности отсчётов имеют место равенства

$$
f_{\ni 1}\left(X_{t}, t^{*}\right)=f_{\ni 1}\left(X^{*}, t\right), \quad f_{\ni 2}\left(X_{t}, t^{*}\right)=f_{\ni 2}\left(X^{*}, t\right) .
$$

Чтобы поддерживать соблюдение этих равенств, следует осуществлять компенсационное изменение величины $X$ сравнительно с ее исходным значением $X^{*}$, получая соответствующие приращения значений первой и второй функций ЭО:

$$
\triangle f_{\ni 1}\left(X_{t}, t^{*}\right)=\triangle f_{\ni 1}\left(X^{*}, t\right), \quad \triangle f_{\ni 2}\left(X_{t}, t^{*}\right)=\triangle f_{\ni 2}\left(X^{*}, t\right) .
$$

Для двух случаев такого изменения первой и второй функций с заданными свойствами имеем:

$$
\begin{aligned}
& \triangle f_{\ni 1}\left(X_{t}, t^{*}\right)=\left.\sum_{\ell=\ell_{1}\left(X^{*}, t^{*}\right)}^{\ell_{1}\left(X_{t}, t^{*}\right)-1} f_{\ni 1 X}^{\prime}\right|_{t^{*}} \cdot \Delta X_{1 l}>0 ; \\
& \triangle f_{\ni 2}\left(X_{t}, t^{*}\right)=\left.\sum_{\ell=\ell_{2}\left(X^{*}, t^{*}\right)}^{\ell_{2}\left(X_{t}, t^{*}\right)-1} f_{\ni 2 X}^{\prime}\right|_{t^{*}} \cdot \Delta X_{2 l}>0 .
\end{aligned}
$$


Учитывая исходно заданные знаки разностных аналогов производных, входящих в алгебраические комбинации (1)-(4), при любых конечных уровнях данных разностных аналогов можно заметить явное противоречие друг другу неравенств (2), (4). Примем теперь за исходный уровень аргумента $t$ произвольное значение $t_{0} \in\left[t^{*}, t\right]$. Тогда, заменяя верхний индекс $*$ на 0 в обозначении $t$, легко видеть, что формулы (1)-(4) сохраняют свою истинность, а замеченное ранее противоречие знаков также сохраняется.

Следовательно, обнаруженное противоречие, которое связано со знаком изменения величины $X$, остается действительным для любого $t_{0}$ из интервала $\left[t^{*}, t\right]$. Поскольку же значения $t^{*}, X^{*}$ в пределах своих диапазонов являются произвольными, то доказанная единственность $X^{*}$, связанная с отсчётами $f_{\ni 1}\left(X^{*}, t_{0}\right), f_{\ni 2}\left(X^{*}, t_{0}\right)$, может быть отнесена и к совокупности этих диапазонов. Итак, наличие обнаруженного противоречия, распространенного на любые значения $X \in D_{X}, t \in D_{t}$, позволяет считать рассмотренное утверждение справедливым.

Нетрудно убедиться в том, что доказанное утверждение сохраняет справедливость и в ситуации следующей модификации знаков разностных аналогов производных:

$$
\left.f_{\ni j X}^{\prime}\right|_{t^{*}}>0,\left.f_{\ni 1 t}^{\prime}\right|_{X^{*}}<0,\left.f_{\ni 2 t}^{\prime}\right|_{X^{*}}>0, X \in D_{X}, t^{*} \in D_{t} .
$$

Вполне естественно считать функции, рассмотренные в рамках доказанного утверждения, относящимися именно к парным функциям ЭО. Таким образом, имея количественное представление функций ЭО, верный экспертный прогноз, касающийся динамики рыночной стоимости ОбО, а также назначив время его приобретения, организация-разработчик получает возможность произвести количественное определение своих затрат на ОбО и критерия его качества. При этом не возникает ситуация, когда в пределах диапазона $D_{X}$ образуется ряд конкурирующих значений величины $X$, что может привести к выполнению дополнительной экспертизы, связанной с дополнительными затратами, а также к соответствующему затягиванию решения вопроса о приобретении ОбО.

Определение уровня $X$ с помощью известных функций ӘО производится при задании стоимостного уровня ОбО $y_{c} \in D_{y}$, соответствующего ситуации реального повышения или снижения стоимости ОбО сравнительно с ее значением $y$, относящегося к моменту выдачи экспертной информации; либо по избранному уровню $X$ с помощью тех же парных функций ЭО производится количественное определение уровня $y_{c}$.

Используя подобный подход, можно находить уровни критерия качества $X$ и стоимости ОбО также для случаев, когда изменения значений любой из функций вида $f_{\ni j}(X, t)$ связаны с участками разнозначных отношений при $j=1$ и $j=2$. Здесь при смене направлений роста-снижения данной функции ЭО граничное положение этой функции, соответствующее моменту перехода от одного направления к другому, принимается за новое исходное состояние. Применительно к нему эксперной группой выстраивается модифицированный прогноз развития стоимостной рыночной ситуации. Возможность такой прогностической коррекции функций ЭО может быть предусмотрена при документальном (договорном) оформлении порядка взаимодействия организации-заказчика экспертизы и экспертной группы. 


\section{БИБЛИОГРАФИЧЕСКИЙ СПИСОК}

1. Ильин В. А., Садовничий В. А., Сендов Бл. Х. Математический анализ. Т. 1: Начальный курс. М.: МГУ, 1985. 662 с. [Ilyin V.A., Sadovnichiy V.A, Sendov Bl. Kh. Mathematical Analysis. Vol. 1. Moscow: MGU, 1985. 662 pp.]

2. Никонов А. И. Выбор вспомогательных чувствительных элементов для блоков эксплуатационно-стабильных датчиков. Уфа, 1989. 9 с. (Деп. в ВИНИТИ 04.07.89, № 4415-В89.) [Nikonov A. I. The choice of auxiliary sensitive elements for units of operating stable sensors. Ufa, 1989. 9 pp. (Deposited at VINITI 04.07.89, No. 4415-B89.)]

3. Турчак Л. И. Основы численных методов. М.: Наука, 1987. 320 с. [Turchak L. I. Basics of Numerical Method. Moscow: Nauka, 1987. 320 pp.]

Поступила в редакцию 04/IV/2012;

в окончательном варианте $-24 / \mathrm{V} / 2012$.

MSC: 26B40

SAMPLING OF EXPERT ASSESSMENT PAIR FUNCTIONS

\section{Y. Antonov, A.I. Nikonov}

Samara State Technical University,

244, Molodogvardeyskaya st., Samara, 443100, Russia.

E-mails: makcant@yandex.ru, nikonovai@mail.ru

The pair functions, used in relation to the value peer review object, which is involved in the implementation of a manufacturing or research purposes, are under consideration. These functions have a consistent character of increasing with respect to the quality criterion of the object, and form a mathematical model for evaluation. The presence of uniqueness displayed by readout functions of this species under the time factor conditions is proved. Traces of such manifestation are related to the assignment of certain signs of the private increments of the functions corresponding to the changes in the estimated object quality criterion, as well as the time delay since its acquisition in comparison with the time of issuance of expert information.

Key words: expert evaluation, the object of evaluation, the function of peer review, coordinated function pairs, calculating of values.

Original article submitted 04/IV/2012; revision submitted $24 / \mathrm{V} / 2012$.

Maxim Yu. Antonov, Postgraduate Studen, Dept. of Electronic Systems and Information Security. Alexander I. Nikonov (Dr. Sci. (Tech.)), Professor, Dept. of Electronic Systems and Information Security. 